\title{
Crystal structure of a secreted lipase from Gibberella zeae reveals a novel "double-lock" mechanism
}

\author{
Zhiyong Lou' ${ }^{1}$, Ming Li ${ }^{2}$, Yuna Sun ${ }^{3}$, Ye Liu ${ }^{2}$, Zheng Liư ${ }^{2}$, Wenping $\mathrm{Wu}^{2}$, Zihe Rao ${ }^{1,3} \bowtie$ \\ ${ }^{1}$ Laboratory of Structural Biology, Tsinghua University, Beijing 100084, China \\ 2 Novozymes China, 14 Xinxi Lu, Shangdi Zone, Haidian District, Beijing 100085, China \\ ${ }^{3}$ National Laboratory of Biomacromolecules, Institute of Biophysics, Chinese Academy of Sciences, Beijing 100101, China \\ $\triangle$ Correspondence: raozh@xtal.tsinghua.edu.cn \\ Received July 10, 2010 Accepted July 25, 2010
}

\section{ABSTRACT}

Fusarium graminearum (sexual stage: Gibberella zeae) is the causative agent of Fusarium Head Blight (FHB), which is one of the most destructive plant disease of cereals, accounting for high grain yield losses, especially for wheat and maize. Like other fungal pathogens, several extracellular enzymes secreted by $G$. zeae are known to be involved in host infection. Among these secreted lipases, G. zeae lipase (GZEL), which is encoded by the FGL1 gene, was demonstrated to be crucial to $G$. zeae pathogenicity. However, the precise mechanism of GZEL remains unclear due to a lack of detailed structural information. In this study, we report the crystal structure of GZEL at the atomic level. The structure of GZEL displays distinct structural differences compared to reported homologues and indicates a unique "double lock" enzymatic mechanism. To gain insight into substrate/inhibitor recognition, we proposed a model of GZEL in complex with substrate and the lipase inhibitor ebelactone B (based on the reported structures of GZEL homologues), which defines possible substrate binding sites within the catalytic cleft and suggests an "anti sn-l" binding mode. These results pave the way to elucidating the mechanism of GZEL and thus provide clues for the design of anti-FHB inhibitors.

KEYWORDS lipase, Gibberella zeae, crystal structure, mechanism, inhibitor

\section{INTRODUCTION}

Fusarium head blight (FHB) is one of the most destructive and economically important crop diseases. It not only causes yield loss, but the causative agent Fusarium graminearum (sexual stage: Gibberella zeae) also produces a variety of mycotoxins that potentially pose a serious threat to human health and animal production (Voigt et al., 2005). With the increase of global temperatures, FHB has spread worldwide, devastating wheat and barley crops and threatening to the global food supply by significantly reducing kernel quality (Bai and Shaner, 2004). Assessments in nine US states showed losses of $\sim 870$ million US dollars due to FHB infection of wheat and barley from 1998 to 2000 (Nganje et al., 2001).

Recently, several extracellular lipases secreted by $G$. zeae (Voigt et al., 2005) were reported to account for its pathogenicity. G. zeae lipase (GZEL), which is one of these secreted lipases, is encoded by the FGL1 gene and was demonstrated to be a virulence factor (Voigt et al., 2005). It is also known that GZEL can be repressed by glucose and inhibited by ebelactone B, a well-known lipase inhibitor (Voigt et al., 2005).

Lipases (triacylglycerol acylhydrolases, EC3.1.1.3) catalyze the hydrolysis of long-chain triglycerides into fatty acids and glycerol and are classified as serine hydrolases due to their inhibition by dirthylp-nitrophenyl phosphate (Hasan et al., 2009). They also represent one of the most important biocatalyst enzyme families and have broad biotechnological applications, such as detergent industry, food production, and pharmaceuticals (Jaeger and Reetz, 1998). Previous results 
demonstrate that lipase activity is greatly increased at the lipid-water interface (i.e., interfacial activation) (Sarda and Desnuelle, 1958). Structural analyses of lipases have revealed that they share a typical $\alpha / \beta$ hydrolase scaffold comprising a core of five (or more) parallel $\beta$-strands flanked by several $\alpha$-helices; their catalytic centers are formed by a highly conserved, trypsin-like, Ser-His-Asp-(Glu) motif (Ollis et al., 1992). Another distinct structural feature of the majority of lipases is the shielding of the catalytic site from the external environment by loops or helices (variously referred to as "loops", "flaps", or (more often) "lids"), which lay over the catalytic center (Brzozowski et al., 2000). According to the position of the "lids", the reported lipase crystal structures are divided into three groups: (i) lipases with lids in a closed position (Brady et al., 1990; Winkler et al., 1990; Schrag et al., 1991); (ii) lipases with open lids stabilized by a covalently bound inhibitor or detergent occluding the active site (Brzozowski et al., 1991; van Tilbeurgh et al., 1993; Grochulski et al., 1994); and (iii) uninhibited lipases with open lids that are not stabilized directly by any substrate or product analog (Schrag et al., 1997).

Here, we report the crystal structure the secreted G. zeae GZEL lipase. The unique C-terminal helix of GZEL, compared to the other reported lipases, prompted us to propose a novel "double lock" enzyme mechanism for GZEL. To gain insight into substrate/inhibitor recognition, we also propose a model of GZEL in complex with substrate and the lipase inhibitor ebelactone $B$ that reveals well-defined sites within the catalytic cleft and suggests an interesting "anti sn-l" binding mode. These results shed light on the unique enzymatic mechanism of GZEL and provide a structural basis for antiFHB inhibitor discovery.

\section{RESULTS AND DISCUSSION}

\section{Overall structure of GZEL}

The cDNA encoding full-length GZEL was cloned and amplified using PCR. The crystal structure consists of amino acids Met1-Ser319, with residues Gly240-Ser259 missing due to a lack of electron density that was likely caused by their flexibility in the structure. The entire GZEL molecule displays a compact globular 3D structure, with a relatively separated $\alpha$-helix ( $\alpha 9$ ), encompassing $50 \times 40 \times$ $40 \AA$ (Fig. 1A and 1B). Though this accessory $\alpha$-helix is quite unique in the lipase family, the GZEL core shares the typical $\alpha / \beta$-hydrolase fold (consisting of a ten-stranded $\beta$-sheet core surrounded eight $\alpha$-helices) reported for other reported lipase structures (Ollis et al., 1992).

The anti-parallel eight strands $(\beta 1-\beta 3$ and $\beta 6-\beta 10)$ in the core part are assembled as a semi-barrel $\beta$-sheet, which is surrounded by five helices ( $\alpha 1-\alpha 2$ and $\alpha 4-\alpha 6$ ) to form the main portion and catalytic center of GZEL. The interface between the core and the $\mathrm{C}$-terminal accessory $\alpha$-helix $\alpha 9$ buries a solvent-accessible surface (SAS) (calculated by using the CCP4 package (Collaborative Computational Project, 1994)) of $860 \AA^{2}$ compared to the $3139 \AA^{2}$ total area of $\alpha 9$, suggesting a strong interaction. The potential interaction surface between the GZEL core and $\alpha 9$ also suggest that there is a positively charged deep groove at the bottom of core, which is fully occupied by $\alpha 9$ in the GZEL structure (Fig. 1C). Compared with previously reported crystal structures of members of the lipase family (Brady et al., 1990; Winkler et al., 1990; Kohno et al., 1996; McAuley et al., 2004), this unique structural feature distinguishes GZEL from other lipases and indicates a putatively different enzymatic mechanism for GZEL.

\section{An unique "lockpin-hinge-lockhole" architecture for GZEL activity}

The GZEL active site is composed of Ser144, Asp198 and His257, which form the conserved catalytic triad reported in other lipase structures. Many factors have been suggested to trigger the activation of lipases at a lipid-water interface (Brockman et al., 1973) and induce alterations of the "lid" conformation from closed to open. The "lid" of the GZEL active site, which is located at $\alpha 3$ helix and arranged similarly to other reported lipases, is formed by residues lle83 to Phe94 (Fig. 3).

Using multiple sequence alignments and DALI searches, several putative key residues in the GZEL active site, both in the primary sequence and in the crystal structure, were identified as highly conserved in lipases from several different species, suggesting a similar enzymatic mechanism for GZEL (Fig. 2). To investigate this mechanism, several homologous lipase structures (Derewenda et al., 1994; Kohno et al., 1996; Brzozowski et al., 2000; McAuley et al., 2004) were carefully superimposed onto the GZEL structure based on their amino acid sequences and structural similarities. We found that the Ser144, Asp198 and His257 residues of the GZEL overlapped quite nicely onto the key residues of homologous lipase active sites with a r.m.s deviation for $\mathrm{C} \alpha$ of $0.5 \AA$. This indicates that, as reported for other catalytic machines, the relative positions of the key active site residues are much better conserved than the general or local protein folds (Fig. 2). Because GZEL was demonstrated to possess lipase activity (Voigt et al., 2005), it is likely that $\alpha 3$, i.e., the lid, must adopt two conformations to fit its different activity states.

Although attempts to crystallize GZEL in an "open-lid" conformation failed, the conservation of GZEL among lipase family members allowed us to predict an open-lid GZEL structure by comparing the experimental lid-closed conformation to the open-lid $T$. lanuginosa lipase structure (PDB code: 1EIN) (Brzozowski et al., 2000) (Fig. 3). The proposed "openlid" conformation was validated by PROCHECK (Laskowski et al., 1993), and the modeled residues (lle83-Phe94) are all in the most favored area (data not shown). The "open and 
A

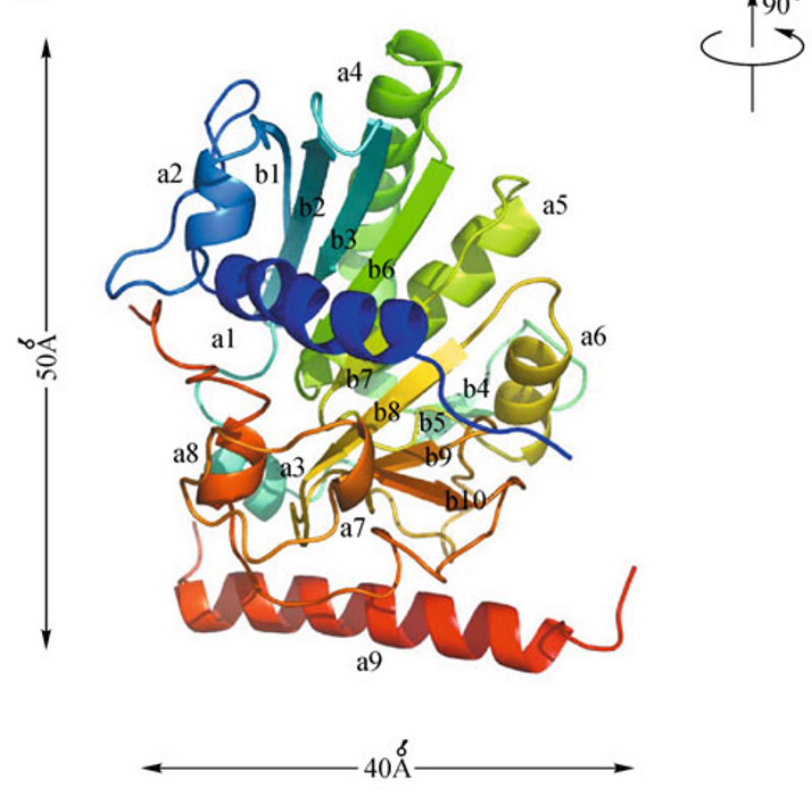

B

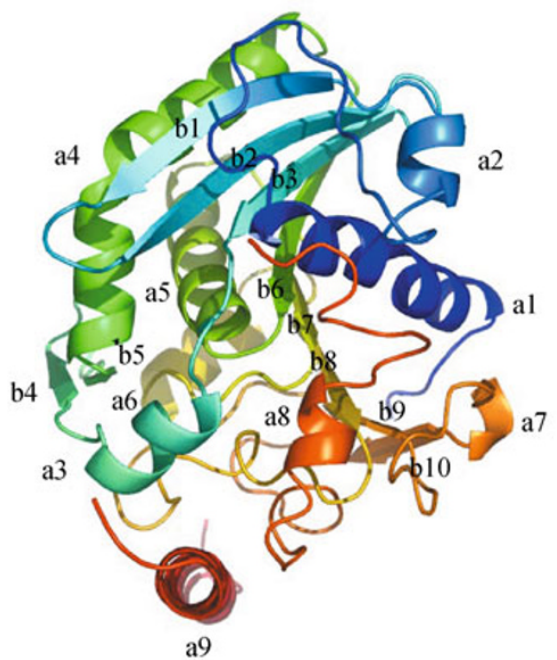

C

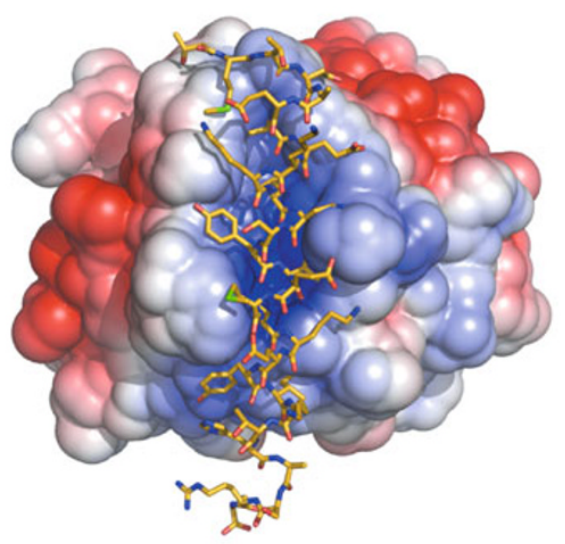

Figure 1. The overall structure of Gibberella zeae lipase. The structure of $G$. zeae lipase is represented in rainbow ribbon format; $\alpha$-helices and $\beta$ strands are labeled as a1-a9 and b1-b10, respectively. (A) Side view of $G$. zeae lipase. (B) View rotated $90^{\circ}$ with respect to A. (C) Potential interaction surface between the enzyme core and $\alpha 9$. The core is shown as a potential surface, and $\alpha 9$ is shown as a golden stick.

closed lid structures show that the lid helix, which is formed by residues lle83 to Phe94, is translated $\sim 7 \AA$ and rotated $\sim 170^{\circ}$ about an axis nearly parallel to that of the helix, which causes it shift away from the closed conformation to expose the active site (Fig. 3A and 3B). Moreover, the hydrophilic side of the lid fragment became partially buried in a polar cavity, which is filled by well-order solvent molecules in the closed form. Therefore, the exposure of the catalytic center is accompanied by a marked increase in the nonpolarity of the surrounding surface. Interfacial activation is thus explained by the stabilization of this nonpolar surface by the lipid environment, which would (in effect) create a catalytically competent enzyme able to attack the triglyceride molecules in the lipid phase (Brzozowski et al., 1991).

By comparison to GZEL homologs, the GZEL crystal structure displays a unique "lockpin-hinge-lockhole" configuration, which is distinctly different from the other lipase or esterase "hinge" structures due to the additional $\alpha 9$ helix. 

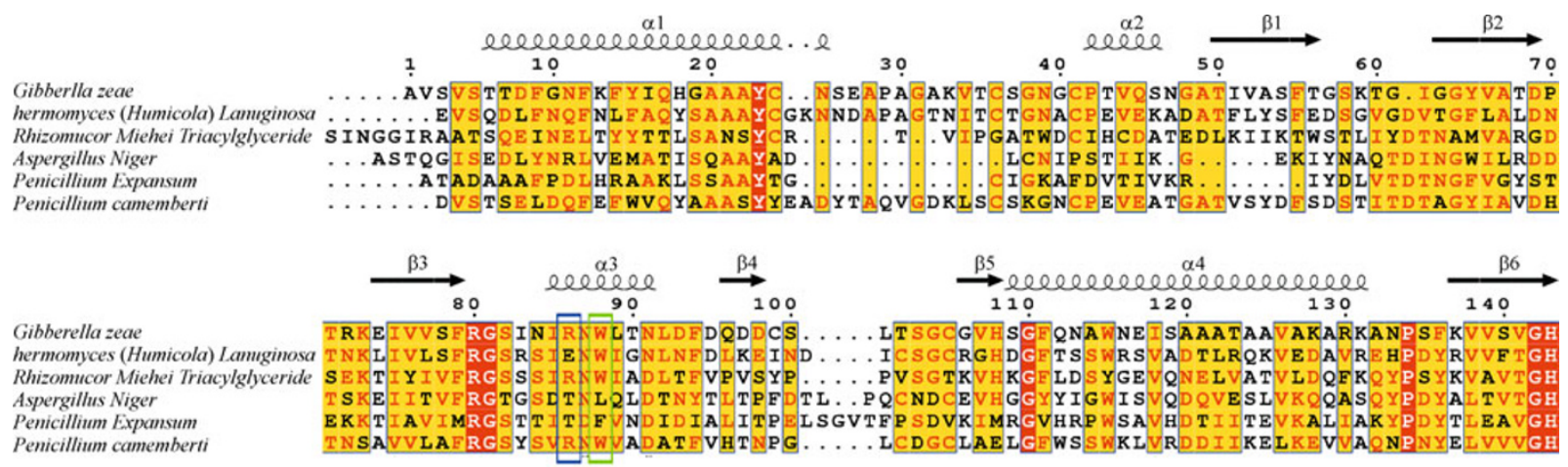

Gibberella zeae
hermomyces (Humicola) Lamuginosa
Rhizomucor Miehei Triacylglyceride
Aspergillus Niger
Penicillium Expansum
Penicillium camemberti
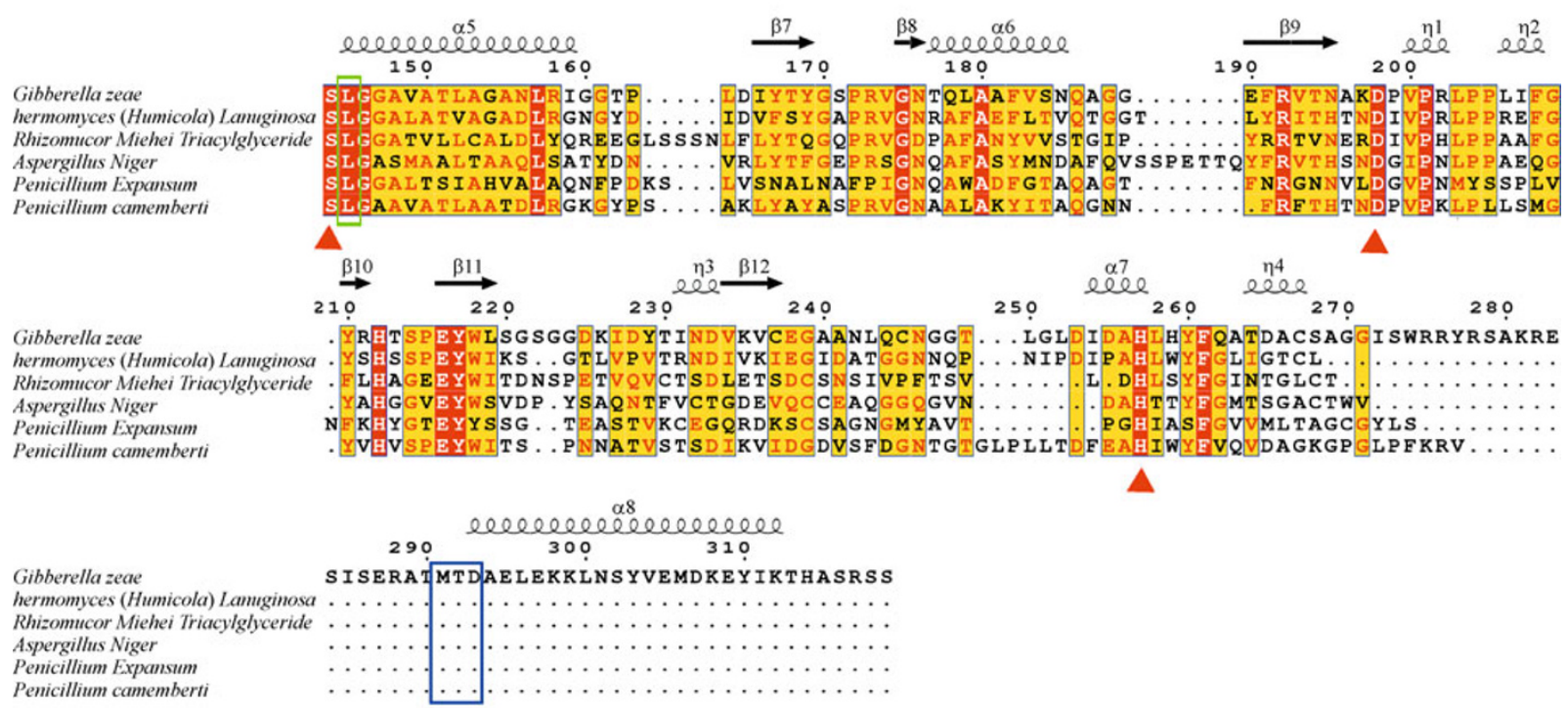

Figure 2. Multiple-sequence alignment of G. zeae lipase with homologs from other organisms. Secondary structural elements of the $G$. zeae lipase crystal structure are shown at the top of the alignment. Arrows indicate $\beta$-strands, and helical curves denote $\alpha$ - or 310-helices. Residues highlighted in red are identical among the compared proteins; residues highlighted in yellow are conserved. The three putative active site residues are marked with red triangles. Residues missing from the crystal structure are underlined. Residues which form the four disulfide bonds are labeled by the underlined green numbers, respectively. The lock and secondary lock residues are highlighted by blue and green frames, respectively.

Generally, there are two major types of "hinge" residues in the lipase family: (i) an Arg in the hinge position, which is present in the T. lanuginosa lipase (Brzozowski et al., 2000; Yapoudjian et al., 2002); and (ii) a Gly in the hinge position, which is found in feruloyl esterases from Aspergillus niger. In sharp contrast, however, the crystal structure of GZEL shows a novel conformational switch configuration. In the lid-closed conformation, two amino nitrogen atoms from Arg86 are hydrogen bonded with the $\mathrm{O}^{\delta 1}$ atom of Asp193 and the carbon atom of Met191, with distances of 2.59 and $2.64 \AA$, respectively. Thus, Arg86 acts as a "lockpin" in this conformation, which is locked by the "lockhole" formed by residues Met191 to Asp193 (Fig. 4A). Furthermore, when in the fully lid-open conformation, the two hydrogen bonds formed by Arg116 and Asp192 with Met191 are broken, and the Arg86 lockpin is released. Therefore, Arg86 turns toward the solution, and the lid assumes a fully open conformation (Fig. 4B).

To analyze the GZEL substrate binding mode, the partial coordinates of oleic acid (OA), which exclude the last seven methyls (referred to as $\mathrm{OA} \triangle 11$ herein) from the previously reported $T$. lanuginose lipase and oleic acid (TLL-OA) complex structure (Yapoudjian et al., 2002), were applied by AUTODOCK (Huey et al., 2007) to calculate the possible binding solution (Fig. 5A and 5B). The final solution, with 186 hits over 250 runs (compared to the second solution with 64 hits), has a free energy of binding of $-4.41 \mathrm{kcal} / \mathrm{mol}$.

The crystal structure of the TLL lipase (PDB code: 1GT6) was superimposed onto the GZEL structure with a r.m.s. deviation of $\mathrm{Ca}$ of $2.36 \AA \hat{\text {. }}$. The comparison of substrate 
A

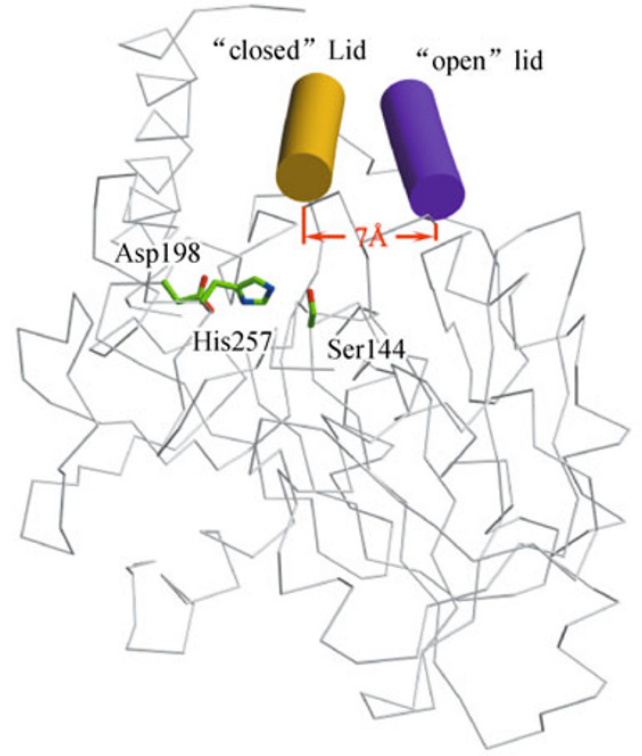

B

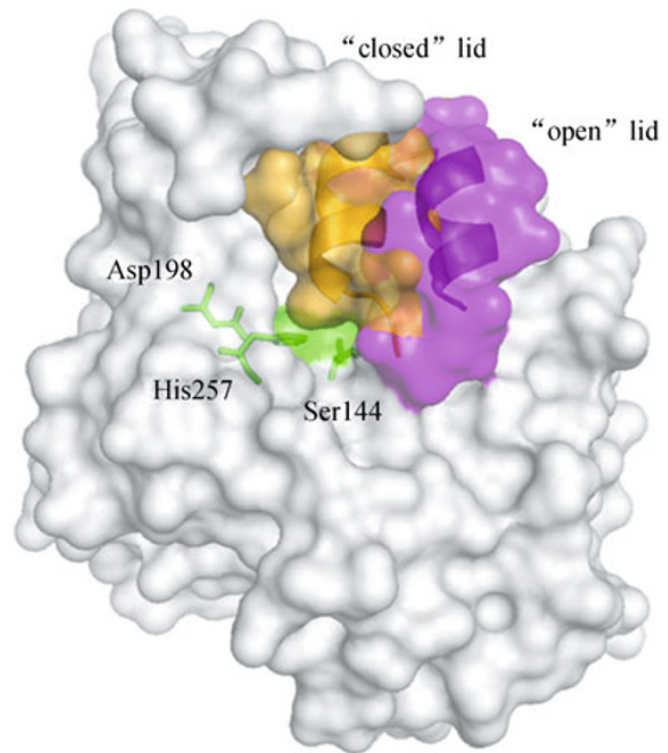

Figure 3. Comparison between the "open" and "closed" lid conformations. (A) The "closed" and "open" lids are represented by gold and purple cylinders, respectively; the same structures are drawn as white lines. The putative key residues are shown as green sticks. (B) Surface representation of the same view in A. The surfaces were colored with the scheme as in A. Both images were produced with Pymol (DeLano, 2002).

A

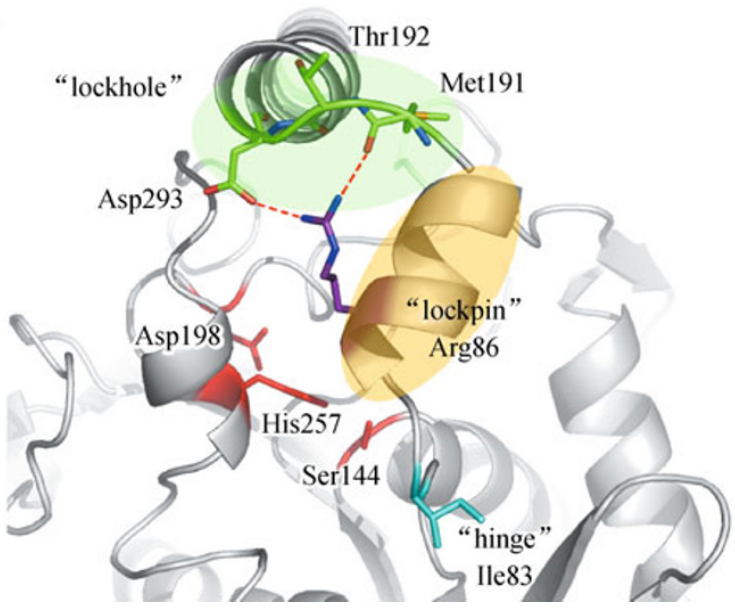

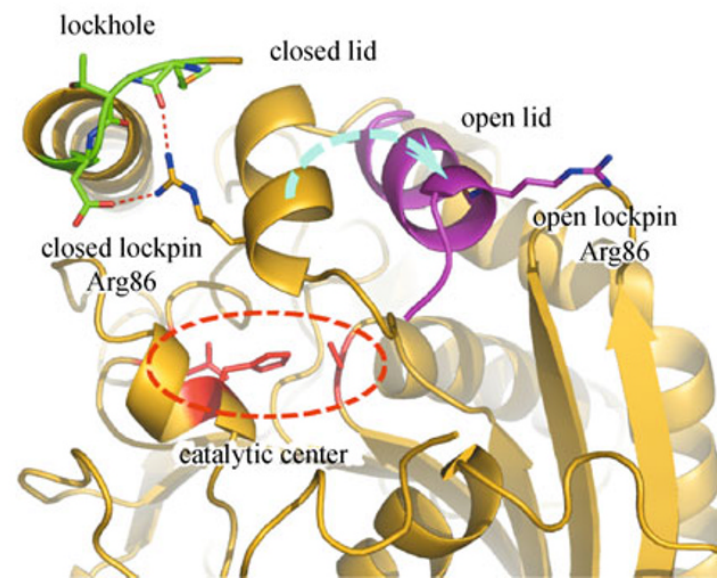

Figure 4. Unique "lockpin-hinge-lockhole" structure. (A) The entire molecule is drawn as a gray ribbon. The "lockhole" is shown as a green stick, the Arg116 "lockpin" is shown as a purple stick, and the lle113 "hinge" is shown as a cyan stick. The key active site residues are red. The hydrogen bonds that lock the lid (shadowed as a golden ellipse) are shown as red, dashed lines. (B) Comparison of the closed and open lockpin.

binding behaviors in TLL and GZEL reveals a relatively similar binding mode. The opened lid in GZEL (lle83-Phe94) and its counterpart (Asp198-lle207) form the left and right walls of the substrate binding cavity, respectively. The side chains of Tyr89-Asn176 and Val107-Gly110 form the bottom of the cavity, with the catalytic center (Ser144-His257) at its top.
This substrate binding cavity is $8 \AA$ in width, $12 \AA$ in height, and displays electron neutrality (Fig. 5C and 5D). The bound OA $\Delta 11$ is deeply trapped in the binding groove with its carboxylic group lying at the top of the binding cavity, which is stabilized by hydrogen bonds with the $\mathrm{N}^{\delta 1}$ atom of Asn81 $(3.2 \AA)$, the $\mathrm{N}^{\varepsilon 2}$ atom of $\mathrm{H} 257(3.9 \AA)$, and the amino atom of 
A<smiles>CCCCCCCCCCC(O)O</smiles>

C

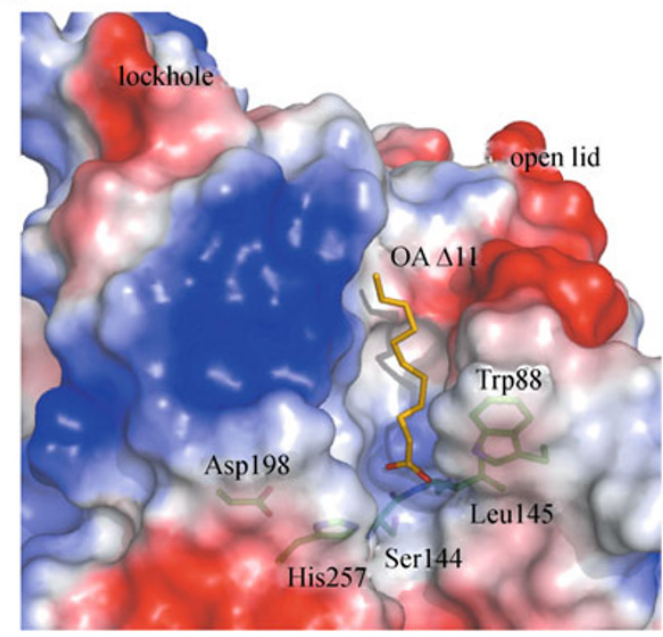

B

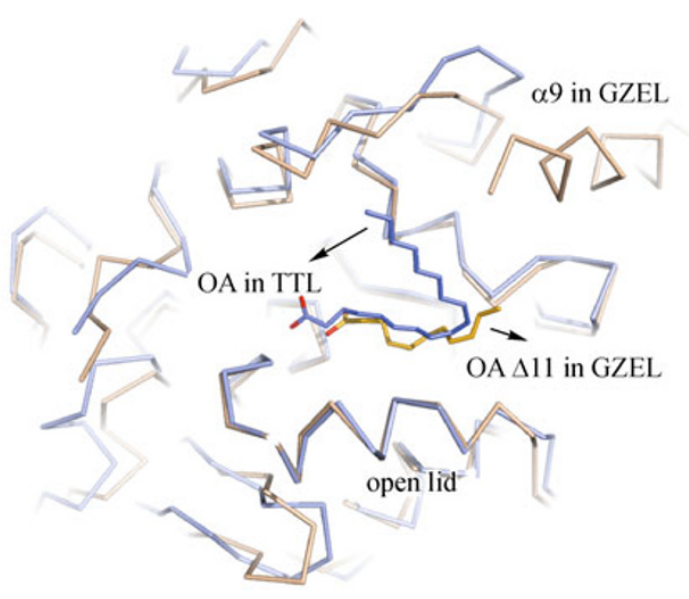

D

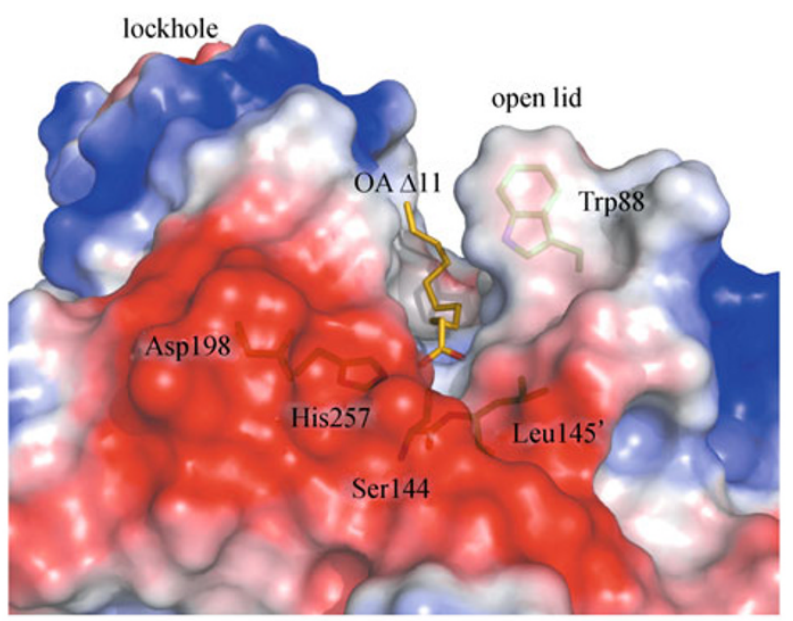

E

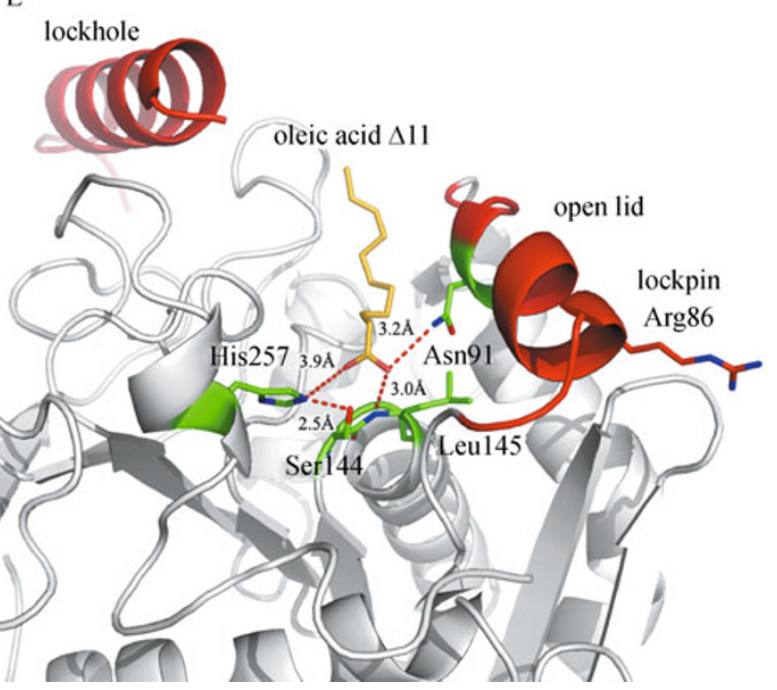

Figure 5. Substrate analog binding behavior. (A) Oleic acid structure. (B) Comparison of the oleic acid binding mode in the Thermomyces (Humicola) lanuginose and $G$. zeae lipases. (C and D) Oleic Acid $\Delta 11$ binding pocket. The bound substrate is drawn as a gold stick, the $G$. zeae lipase is shown as a semitransparent potential surface, and the key residues important for substrate binding and enzyme activity are shown as green sticks. (C) Top view of the binding pocket. (D) Side view of the binding pocket. (E) $G$. zeae lipase bound to oleic acid $\Delta 11$. Bound oleic acid $\Delta 11$ and key residues were shown as gold and green sticks, respectively. The lockhole-lockpin and open lid are highlighted, and the possible hydrogen bonds and their distances are shown. 
Leu145 (3.0 $\AA)$. The fact that the alkyl chains of OA $\Delta 11$ occupy the sn-1 position in the active site indicates that the lipid binds according to the conventional mode in GZEL.

In the catalytic center, His257 occupies a position where the $\mathrm{N}^{\varepsilon}$ atom forms a hydrogen bond to the $\mathrm{O}^{\mathrm{V}}$ atom of Ser134 (distance of $2.5 \AA$ ), and the $\mathrm{N}^{\bar{\delta}}$ atom hydrogen bonds to Asp198 (distances of $2.6 \AA$ to $\mathrm{O}^{\delta 1}$ and $3.5 \AA$ to $\mathrm{O}^{\delta 2}$ ). This arrangement of residues is characteristic of enzymes that cleave ester bonds by nucleophilic attack and is known as a catalytic triad (Hedstrom, 2002). His 257 acts as a general base by deprotonating the serine, which allows nucleophilic attack of the carbonyl $\mathrm{C}$ atom of the substrate by the serine, yielding a tetrahedral transition state. The resulting positive charge on the histidine is stabilized by an electrostatic interaction with the negatively charged aspartic acid residue. The tetrahedral transition state collapses to give an acylenzyme intermediate. The next step is an attack of the acylenzyme by a nucleophilic water molecule, again activated by the histidine residue, leading to release of the acid product via a second tetrahedral intermediate transition state. The negative charge on the carbonyl $\mathrm{O}$ atom of the tetrahedral intermediates is stabilized by the oxyanion hole formed by the backbone $\mathrm{N}$ atoms of (in this case) residues Ser82 and Leu145.

These results suggest that the conformation of the GZEL lid may first change by interfacial activation, and then the lipase bond is subsequently hydrolyzed by a mechanism very similar to that used by serine proteases. Additionally, the lid may also serve as a device to inhibit the proteolytic activity of the triad, thereby protecting the enzyme itself.

\section{Accessory lock in the lipase family}

Combing the structural and functional results, Trp88 and Leu145 are indicated to be crucial for substrate recognition and binding, acting as an accessory and small "lockholelockpin." In the lid-closed conformation, Trp88 is located near Leu145 (3.6 $\AA$ away), which indicates a relatively strong Van der Waals interaction that may seal the top of the binding cavity like an accessory lock (Fig. 6). In contrast, this accessory lock is released (moved a distance of nearly $8 \AA$ ) and can accommodate the carboxylic group of bound substrate in the lid-open conformation. By comparing the sequences of GZEL homologs and their structures, a similar "accessory lock" also appears in other lipases. In the multiple sequence alignment, we show that Leu145 is conserved in all G. zeae lipase homologs, while Trp88 is also largely conserved in these homologs, though identically replaced by leucine in the Aspergillus oryzae, A. niger and Feruloyl esterases. The only exception is Rhiropus niveus, which has an alanine residue instead of a Trp or Leu. All of these lipase structures were superimposed (Fig. 6B), and we found that the position of Trp88 and Leu145 were also structurally conserved. The Leu145 position is only in the locked form in the $R$. niveus lipase (RNL) and the closed conformation of GZEL, which is consistent with the sequence alignment results. Related substrate molecular docking was applied to $\mathrm{OA} \triangle 11$ with different Leu145 conformations in the GZEL open conformation. From this comparison, it can be seen that bound OA $\triangle 11$ moves out of the substrate binding cavity following the conformational change of the Leu 145 side chain. All of these facts suggest that this "accessory lock" may exist universally in the lipase family and be crucial for substrate binding.

\section{Possible substrate/inhibitor binding}

Because the activity of GZEL can be inhibited by ebelactone B (Fig. 7A) both in vivo and in vitro (Voigt et al., 2005), we attempted to simulate the enzyme-inhibitor complex on the open-lid conformation to explain a possible inhibition mechanism (Fig. 7). We performed the molecular docking in AUTODOCK4 (Huey et al., 2007); the best solution (53 hits in $250 \mathrm{runs}$ ) had a $-8.42 \mathrm{kcal} / \mathrm{mol}$ free energy and was used to investigate the inhibition.

The inhibitor binding model shows that ebelactone $B$ binds to GZEL with an interesting "anti sn-1" binding mode, which was reported previously (Yapoudjian et al., 2002) (Fig. 7B and 7C). Through examining the structures of ebelactone $B$ and oleic acid, we found that the tail of ebelactone $B$ is structurally similar to the carboxylic group of oleic acid. The last methyl occupies the carboxylic group binding position, with distances of 3.2 and $3.5 \AA$ away from the $\mathrm{O}^{\vee}$ atom of Ser144 and the $\mathrm{N}^{\delta}$ atom of His257, respectively. The top tetra-lactonic group of ebelactone $B$ is stabilized by the end of the GZEL substrate binding cavity via hydrophobic interactions. In addition, the phenyl ring of Phe94, which is parallel to the tetra-lactonic group, also stabilizes ebelactone $B$ by a conjugated effect (Fig. 7C). These facts suggest that ebelactone $B$ stably binds to the catalytic cavity of GZEL, which is consistent with biochemistry investigations (Voigt et al., 2005).

\section{CONCLUSIONS}

In this study, we reported the crystal structure of GZEL with a novel "lockhole-hinge-lockpin" enzyme activity switch. Multiple sequence alignments and structure comparisons with lipases/esterases from different species show that GZEL has a classic $\alpha / \beta$-hydrolase fold and highly conserved key residues in its active site, which indicate that GZEL functions in a similar manner as other lipases/esterases. Structural analysis revealed that the lockhole-hinge-lockpin" activity switch is formed by an accessory C-terminal $\alpha$-helix, as well as Arg86 and lle83 in the lid helix, and is unique in all reported lipase/esterase structures. Furthermore, the models of GZEL in complex with substrate and inhibitor indicate that residues Arg86 to Leu145 (or their counterparts in homologs) should function as an accessory lock in most of the reported lipase 


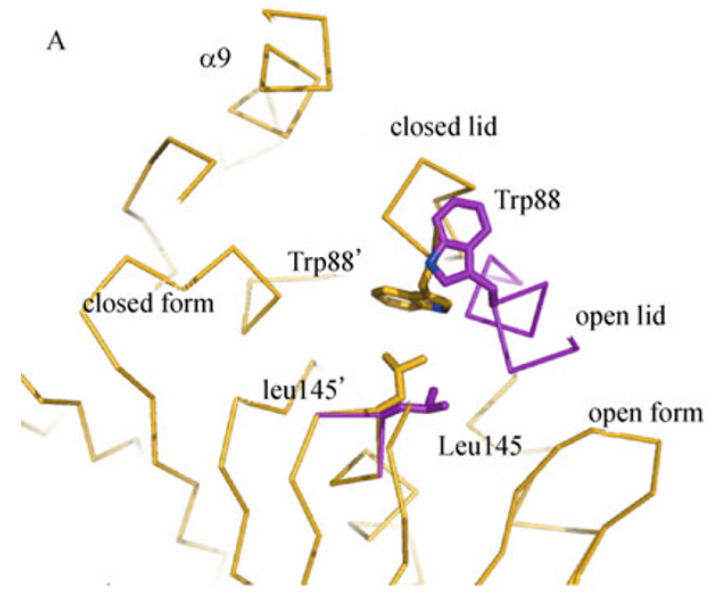

B

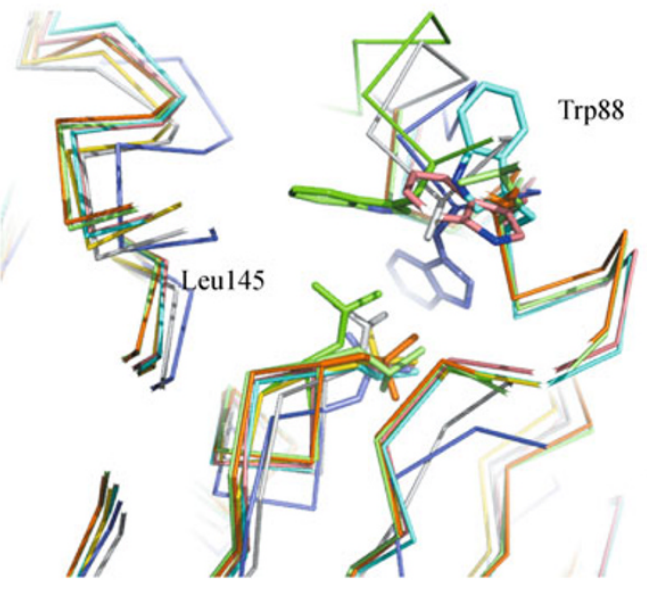

C

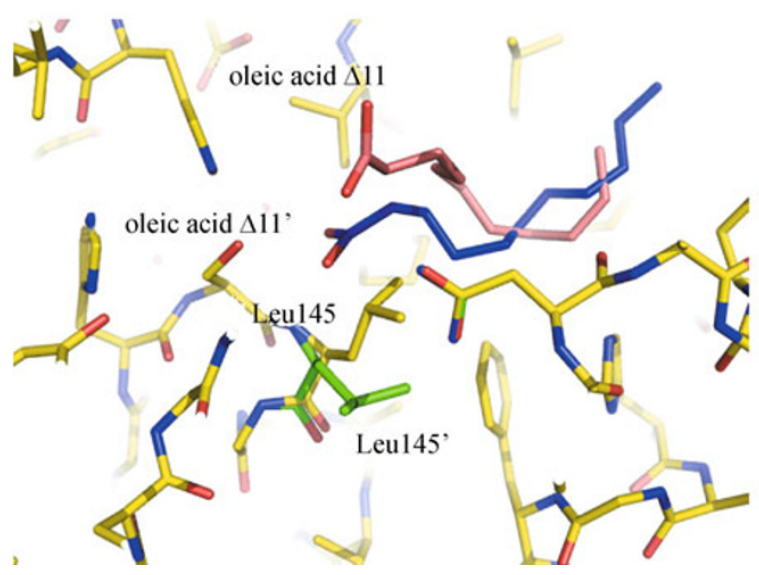

Figure 6. Key role of the secondary lock. (A) Comparison of Trp118-Leu175 in the G. zeae lipase open and closed conformations. The molecules are drawn as ribbons; the secondary locks in the closed and open conformations are shown as purple and gold sticks, respectively. (B) Superposition of the secondary lock in lipases from other species. (C) Conformational comparison of oleic acid $\Delta 11$ in the open and closed conformations of $G$. zeae lipase. The enzyme and substrate analog are shown as gold and purple sticks, respectively, in the Leu175-locked conformation and green and blue sticks, respectively, in the Leu175-released conformation.

structures. Hence, we purpose that enzymes such as GZEL utilize a "double lock" mechanism. The molecular docking of a known inhibitor (Voigt et al., 2005) also supports this model.

\section{MATERIALS AND METHODS}

\section{Protein expression, purification, and crystallization}

The FGL1 gene was amplified by PCR, and the GZEL protein was overexpressed and subsequently purified via a previously reported method (Voigt et al., 2005) with some modifications. Briefly, the entire FGL1 coding sequence of was PCR-amplified from $F$. graminearum CDNA. Two individual PCR fragments were independently obtained by using two sets of primer pairs: Na (5'-GATCCACCATGCGTCTCC TGTCACTCCTC-3') and Cb (5'-CTGATGAGCGGCTGGCGTGAG3'); and Nb (5'-CACCATGCGTCTCCTGTCACTCCTC-3') and Ca (5'AATTCTGATGAGCGGCTGGCGTGAG-3'). The fragment was then ligated into the same sites in the pLIZG7 expression vector. An EFHHHHHHP tag was fused to the $\mathrm{C}$ terminus of the recombinant protein. Plasmid DNA containing the FGL1 gene was then linearized with Pmel (NEB) and transformed into Pichia pastoris KM71 (Invitrogen) following the manufacturer's instructions. Transformants were grown on a histidine-deficient MD plate for 2-3 d. The positive candidates were inoculated into $3 \mathrm{~mL} \mathrm{BMSY}$, and the cultures were shaken in a $28^{\circ} \mathrm{C}$ incubator for $3 \mathrm{~d}$ until the $\mathrm{OD}_{600}$ reached 20. Methanol was then added daily to the culture to a final concentration of $0.5 \%$ for the next $4 d$ to induce GZEL expression. On day 4 of 
A<smiles>CCC(C)C(O)C(C)C(=O)C(C)C=C(C)CC(C)C1OC(=O)C1CC</smiles>

B

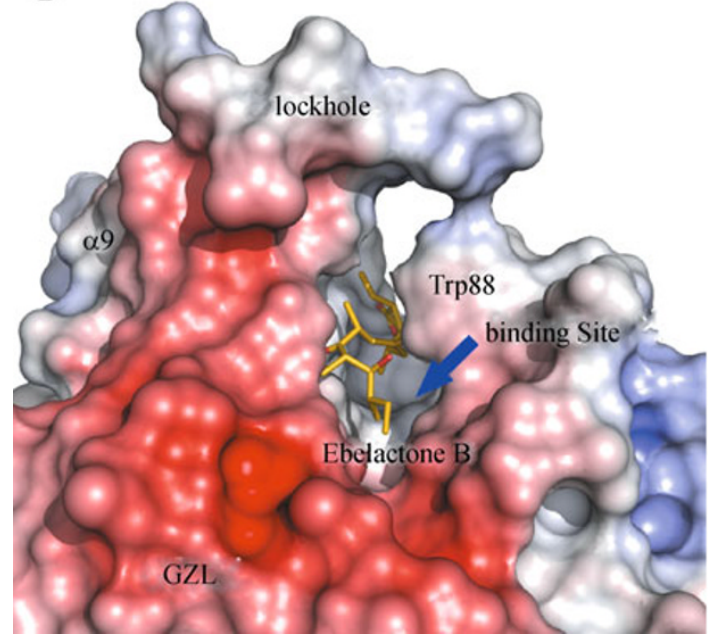

C

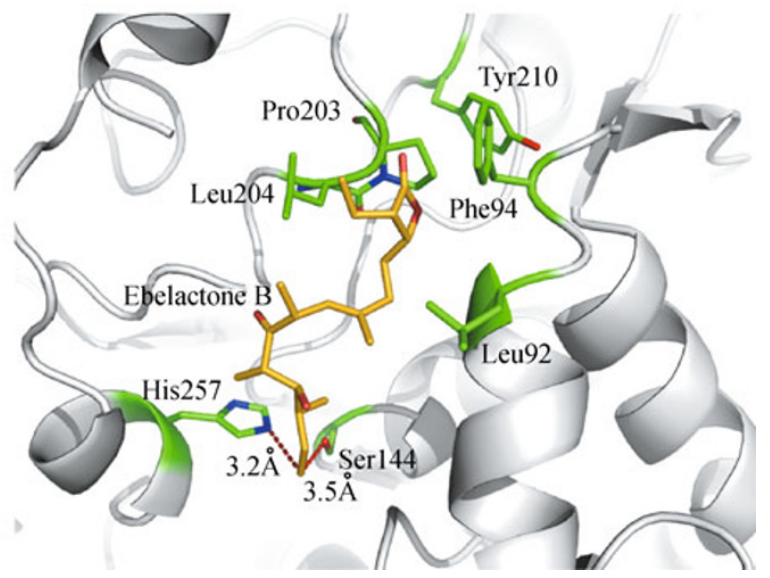

Figure 7. Purposed inhibitor binding model. (A) Structure of ebelactone B. (B) Ebelactone B binding site in GZL. The GZL molecule is shown as a potential surface, and ebelactone $B$ is shown as golden sticks. (C) Details of ebelactone B binding to GZL. Ebelactone B and residues in GZL are shown as gold and green sticks, respectively.

induction, the cultures were harvested by centrifugation, and the activity of the supernatant was tested on olive oil/Bright Green plates $(\mathrm{pH}$ 7.0). The positive clones showed dark green zones. Positive supernatants also showed a protein band of the appropriate size on SDS-PAGE followed by Coomassie staining.

The culture supernatant was separated from the cells by centrifugation, and the $\mathrm{pH}$ of the supernatant was adjusted to 7.0. The supernatant was then filtered and applied onto a Ni-Sepharose FF column equilibrated with $25 \mathrm{mM}$ Tris- $\mathrm{HCl}(\mathrm{pH} 7.0)$ and $0.3 \mathrm{M} \mathrm{NaCl}$. The target protein was eluted with a $0-1 \mathrm{M}$ imidazole gradient, and fractions from the column were analyzed for activity. The fractions displaying peak enzyme activity were pooled and concentrated. The samples were then loaded onto a Superdex 75 gel-filtration column equilibrated with $25 \mathrm{mM}$ Tris- $\mathrm{HCl}(\mathrm{pH} 8.0)$ and $0.15 \mathrm{M} \mathrm{NaCl}$, and the eluted active lipase was concentrated and dialyzed against $25 \mathrm{mM}$ Tris-HCl (pH 8.0). The lipase was analyzed by SDS-PAGE, and pure fractions were prepared for crystallization trials.

Crystallization experiments were conducted using the vapor diffusion method (Sun et al., 2008). Briefly, crystals were grown at $18^{\circ} \mathrm{C}$ from a solution containing $10 \mathrm{mg} / \mathrm{mL}$ protein, $0.2 \mathrm{M}$ ammonium sulfate, 0.1 M Bis-Tris ( $\mathrm{pH} 5.5$ ), and 25\% (w/v) PEG-3350.

\section{Data collection}

The native GZEL data set was collected at $100 \mathrm{~K}$ using an in-house Rigaku MM-007 generator with a Mar345dtb image plate detector. Because the well solution an efficient for cyro-protectant, the fresh crystals were directly flash-cooled in a stream of nitrogen gas cooled to $100 \mathrm{~K}$. The resolution of data extended to $2.8 \AA$, with an overall $R_{\text {merge }}$ of $10.1 \%$. The crystal belongs to space group $P 2_{1} 2_{1} 2_{1}$, with the unit-cell parameters $a=78.4 \AA, b=91.0 \AA, c=195.8 \AA$, and $\alpha=\beta$ $=\gamma=90^{\circ}$. We assumed four GZEL molecules per asymmetric unit with a $V M$ of $2.6 \AA^{3} \mathrm{Da}^{-1}$, corresponding to a solvent content of $48 \%$ (Matthews, 1968). Processing of diffraction images and scaling of the integrated intensities were performed using the HKL2000 software package (Otwinowski and Minor, 1997). The statistics of all data collections are presented in Table 1.

\section{Structure determination and refinement}

The structure of GZEL was solved by molecular replacement, employing the crystal structure of the lipase from Thermomyces lanuginosa (PDBank code: 1EIN) as the initial searching model, by using the program PHASER (McCoy et al., 2005). The clear solutions in both the rotation and translation functions indicated the presence of four molecules in one asymmetric unit, which is consistent with the Matthews coefficient and solvent content (Matthews, 1968). Residues which differ between GZEL and the searching model were manually rebuilt in the program $\mathrm{O}$ (Jones et al., 1991) under the guidance of $F_{O}$ $F C$ and $2 F o-F C$ electron density maps.

After the initial refinement, a distinct density was observed in the Fo-FC map, which should belong to the additional Thr320-Ser349 residues of the GZEL polypeptide. The program DM (Cowtan, 1994) was then used to perform density modification in order to refine the 
Table 1 Data collection and refinement statistics

\begin{tabular}{|c|c|}
\hline & GZEL native data set \\
\hline \multicolumn{2}{|l|}{ data processing } \\
\hline space group & $P 2{ }_{1}{ }_{1} 2_{1}$ \\
\hline \multirow[t]{2}{*}{ cell parameters $\left(\mathrm{A},{ }^{\circ}\right)$} & $a=78.4 \AA, b=91.0 \AA, c=195.8 \AA$ \\
\hline & $\alpha=\beta=\gamma=90^{\circ}$ \\
\hline resolution range $(\AA)$ & $50.0-2.8(2.9-2.8)^{a}$ \\
\hline total reflections & 326,163 \\
\hline unique reflections & 34,080 \\
\hline completeness (\%) & $9.6(8.0)^{a}$ \\
\hline$R_{\text {merge }}(\%)^{\mathrm{b}}$ & $10.1(48.0)^{\mathrm{a}}$ \\
\hline $1 / \sigma$ & $97.7(92.3)^{a}$ \\
\hline \multicolumn{2}{|l|}{ refinement } \\
\hline No. of reflections used & 29217 \\
\hline No. of reflections in test set & 2.906 \\
\hline$R$-factor $(\%)^{\mathrm{c}}$ & 23.5 \\
\hline$R_{\text {free }}(\%)^{\mathrm{C}}$ & 26.4 \\
\hline \multicolumn{2}{|l|}{ r.m.s. deviation } \\
\hline bonds $(\AA)$ & 0.006 \\
\hline angles $\left({ }^{\circ}\right)$ & 1.097 \\
\hline average $B$ factor & 44.8 \\
\hline \multicolumn{2}{|l|}{ ramachandran plot } \\
\hline $\begin{array}{l}\text { residues in most favored } \\
\text { regions }\end{array}$ & 95.4 \\
\hline $\begin{array}{l}\text { residues in additional allowed } \\
\text { regions }\end{array}$ & 4.6 \\
\hline
\end{tabular}

a Numbers in parentheses correspond to the highest resolution shell.

${ }^{\mathrm{b}} R_{\text {merge }}=\sum \mathrm{h} \sum \mathrm{l} \mid$ lih $-<\mathrm{lh}>\mid / \sum \mathrm{h} \sum \mathrm{l}<\mathrm{lh}>$, where $<\mathrm{lh}>$ is the mean intensity of the observations lih of reflection $h$.

${ }^{\mathrm{c}} R$ factor $=\Sigma(||$ Fobs $\mid-$ Fcalc ||$) / \sum \mid$ Fobs $\mid ; R_{\text {free }}$ is the $R$ factor for a subset $(5 \%)$ of reflections that was selected prior to refinement calculations and not included in the refinement.

phases of this difference density and avoid model bias that may have arisen from phasing by molecular replacement. The remaining residues were manually built into their density in the programs $\mathrm{O}$ and COOT (Jones et al., 1991; Emsley and Cowtan, 2004) to generate the final complete coordinates. After the refinement of the model using simulated annealing, energy minimization, restrained individual B factors and addition of 221 water molecules in CNS and PHENIX (Brunger et al., 1998; Adams et al., 2002), the respective working $R$-factor and $R_{\text {free }}$ dropped from 0.42 and 0.45 to 0.23 and 0.26 , respectively, for all data from 50.0 to $2.8 \AA$. Refinement was monitored by calculating $R_{\text {free }}$ based on a subset containing $5 \%$ of the total reflections. Model geometry was verified using the program PROCHECK (Laskowski et al., 1993). Data collection and refinement statistics are detailed in Table 1. All structure figures were prepared using PYMOL (DeLano, 2002).

\section{Computational methods}

GZEL sequence and structural homologs were identified using
BLASTp searches against the sequence database at the National Center for Biotechnology Information, and by DALI searches against the structural database (Holm et al., 2008). A structural multiple sequence alignment of these homologous protein sequences was generated using ClustalX (Thompson et al., 1997) and plotted with ESPript (Gouet et al., 2003). Substrate and inhibitor docking studies were performed using AutoDock4 (Huey et al., 2007) with MGLTools1.4.5.

\section{PROTEIN STRUCTURE ACCESSION NUMBER}

Coordinates and structure factors of the GZEL crystal structure were deposited in the Protein Data Bank under accession number 3NGM.

\section{ACKNOWLEDGEMENTS}

This work was supported by the National Programs for High Technology Research and Development Program of China (863 Program) (Grant No. 2009ZX10004-304), the National Natural Science Foundation of China (NSFC) (Grant No. 30870486), and the National Major Project (Grant Nos. 2009ZX09311-00, 2006AA02A322).

\section{REFERENCES}

Adams, P.D., Grosse-Kunstleve, R.W., Hung, L.W., loerger, T.R., McCoy, A.J., Moriarty, N.W., Read, R.J., Sacchettini, J.C., Sauter, N.K., and Terwilliger, T.C. (2002). PHENIX: building new software for automated crystallographic structure determination. Acta Crystallogr D Biol Crystallogr 58, 1948-1954.

Bai, G., and Shaner, G. (2004). Management and resistance in wheat and barley to fusarium head blight. Annu Rev Phytopathol 42, 135-161.

Brady, L., Brzozowski, A.M., Derewenda, Z.S., Dodson, E., Dodson, G.G., Tolley, S., Turkenburg, J.P., Christiansen, L., Huge-Jensen, B., Norskov, L., et al. (1990). A serine protease triad forms the catalytic centre of a triacylglycerol lipase. Nature 343, 767-770.

Brockman, H.L., Law, J.H., and Kézdy, F.J. (1973). Catalysis by adsorbed enzymes. The hydrolysis of tripropionin by pancreatic lipase adsorbed to siliconized glass beads. J Biol Chem 248, 4965-4970.

Brünger, A.T., Adams, P.D., Clore, G.M., DeLano, W.L., Gros, P., Grosse-Kunstleve, R.W., Jiang, J.S., Kuszewski, J., Nilges, M., Pannu, N.S., et al. (1998). Crystallography \& NMR system: A new software suite for macromolecular structure determination. Acta Crystallogr D Biol Crystallogr 54, 905-921.

Brzozowski, A.M., Derewenda, U., Derewenda, Z.S., Dodson, G.G., Lawson, D.M., Turkenburg, J.P., Bjorkling, F., Huge-Jensen, B., Patkar, S.A., and Thim, L. (1991). A model for interfacial activation in lipases from the structure of a fungal lipase-inhibitor complex. Nature 351, 491-494.

Brzozowski, A.M., Savage, H., Verma, C.S., Turkenburg, J.P., Lawson, D.M., Svendsen, A., and Patkar, S. (2000). Structural origins of the interfacial activation in Thermomyces (Humicola) lanuginosa lipase. Biochemistry 39, 15071-15082.

Collaborative Computational Project, N., 1994. The CCP4 suite: programs for protein crystallography. Acta Crystallogr. Sect. D Biol. Crystallogr.: 760-763. 
Cowtan, K., 1994. Joint CCP4 and ESF-EACBM Newsletter on Protein Crystallography: 34-38.

DeLano, W.L. 2002. The PyMOL Molecular Graphics System World Wide Web http://www.pymol.org.

Derewenda, U., Swenson, L., Green, R., Wei, Y., Dodson, G.G., Yamaguchi, S., Haas, M.J., and Derewenda, Z.S. (1994). An unusual buried polar cluster in a family of fungal lipases. Nat Struct Biol 1, 36-47.

Emsley, P., and Cowtan, K. (2004). Coot: model-building tools for molecular graphics. Acta Crystallogr D Biol Crystallogr 60, 2126-2132.

Gouet, P., Robert, X., and Courcelle, E. (2003). ESPript/ENDscript: Extracting and rendering sequence and $3 \mathrm{D}$ information from atomic structures of proteins. Nucleic Acids Res 31, 3320-3323.

Grochulski, P., Bouthillier, F., Kazlauskas, R.J., Serreqi, A.N., Schrag, J.D., Ziomek, E., and Cygler, M. (1994). Analogs of reaction intermediates identify a unique substrate binding site in Candida rugosa lipase. Biochemistry 33, 3494-3500.

Hasan, F., Shah, A.A., and Hameed, A. (2009). Methods for detection and characterization of lipases: A comprehensive review. Biotechnol Adv 27, 782-798.

Hedstrom, L. (2002). Serine protease mechanism and specificity. Chem Rev 102, 4501-4524.

Holm, L., Kääriäinen, S., Rosenström, P., and Schenkel, A. (2008). Searching protein structure databases with DaliLite v.3. Bioinformatics 24, 2780-2781.

Huey, R., Morris, G.M., Olson, A.J., and Goodsell, D.S. (2007). A semiempirical free energy force field with charge-based desolvation. J Comput Chem 28, 1145-1152.

Jaeger, K.E., and Reetz, M.T. (1998). Microbial lipases form versatile tools for biotechnology. Trends Biotechnol 16, 396-403.

Jones, T.A., Zou, J.Y., Cowan, S.W., and Kjeldgaard, M. (1991). Improved methods for building protein models in electron density maps and the location of errors in these models. Acta Crystallogr $A$ 47, 110-119.

Kohno, M., Funatsu, J., Mikami, B., Kugimiya, W., Matsuo, T., and Morita, Y. (1996). The crystal structure of lipase II from Rhizopus niveus at 2.2 A resolution. J Biochem 120, 505-510.

Laskowski, R., MacArthur, M., Moss, D., and Thornton, J. (1993). PROCHECK: a program to check the stereochemical quality of protein structures. J Appl Cryst 26, 283-291.

Matthews, B.W. (1968). Solvent content of protein crystals. J Mol Biol 33, 491-497.

McAuley, K.E., Svendsen, A., Patkar, S.A., and Wilson, K.S. (2004). Structure of a feruloyl esterase from Aspergillus niger. Acta
Crystallogr D Biol Crystallogr 60, 878-887.

McCoy, A.J., Grosse-Kunstleve, R.W., Storoni, L.C., and Read, R.J. (2005). Likelihood-enhanced fast translation functions. Acta Crystallogr D Biol Crystallogr 61, 458-464.

Nganje, W.E., Johnson, D.D., Wilson, W.W., Leistritz, F.L., Bangsund, D.A., and Tiapo, N.M. 2001. Economic impacts of fusarium head blight in wheat and barley: 1998-2000. In Agribusiness and Applied Economics Report No.464.

Ollis, D.L., Cheah, E., Cygler, M., Dijkstra, B., Frolow, F., Franken, S. M., Harel, M., Remington, S.J., Silman, I., Schrag, J., et al. (1992). The alpha/beta hydrolase fold. Protein Eng 5, 197-211.

Otwinowski, Z., and Minor, W. 1997. Processing of X-ray diffraction data collected in oscillation mode, p. 307-326, in: C. W. Carter Jr. and R. M. Sweet, Eds.), Macromolecular Crystallography, part A, Academic Press.

Sarda, L., and Desnuelle, P. (1958). Actions of pancreatic lipase on esters in emulsions. Biochim Biophys Acta 30, 513-521.

Schrag, J.D., Li, Y.G., Wu, S., and Cygler, M. (1991). Ser-His-Glu triad forms the catalytic site of the lipase from Geotrichum candidum. Nature 351, 761-764.

Schrag, J.D., Li, Y., Cygler, M., Lang, D., Burgdorf, T., Hecht, H.-J., Schmid, R., Schomburg, D., Rydel, T.J., Oliver, J.D., et al. (1997). The open conformation of a Pseudomonas lipase. Structure 5, 187-202.

Sun, Y., Li, M., Zhang, Y., Liu, L., Liu, Y., Liu, Z., Li, X., and Lou, Z. (2008). Crystallization and preliminary crystallographic analysis of Gibberella zeae extracellular lipase. Acta Crystallogr Sect F Struct Biol Cryst Commun 64, 813-815.

Thompson, J.D., Gibson, T.J., Plewniak, F., Jeanmougin, F., and Higgins, D.G. (1997). The CLUSTAL_X windows interface: flexible strategies for multiple sequence alignment aided by quality analysis tools. Nucleic Acids Res 25, 4876-4882.

van Tilbeurgh, H., Egloff, M.-P., Martinez, C., Rugani, N., Verger, R., and Cambillau, C. (1993). Interfacial activation of the lipaseprocolipase complex by mixed micelles revealed by X-ray crystallography. Nature 362, 814-820.

Voigt, C.A., Schäfer, W., and Salomon, S. (2005). A secreted lipase of Fusarium graminearum is a virulence factor required for infection of cereals. Plant J 42, 364-375.

Winkler, F.K., D'Arcy, A., and Hunziker, W. (1990). Structure of human pancreatic lipase. Nature 343, 771-774.

Yapoudjian, S., Ivanova, M.G., Brzozowski, A.M., Patkar, S.A., Vind, J., Svendsen, A., and Verger, R. (2002). Binding of Thermomyces (Humicola) lanuginosa lipase to the mixed micelles of cis-parinaric acid/NaTDC. Eur J Biochem 269, 1613-1621. 